\title{
The principle of "incomplete comprehension of object" in the context of the discourse of uncertainties in a digital society
}

\author{
Olena Polishchuk ${ }^{1, *}$, Nataliia Kovtun ${ }^{1, * *}$, Iryna Vitiuk $^{1, * * *}$, Roman Sapeńko $^{2, * * * *}$, and Bogdan Trocha ${ }^{2, \dagger}$ \\ ${ }^{1}$ Zhytomyr Ivan Franko State University, 40 Velyka Berdychivska Str., Zhytomyr, 10008, Ukraine \\ ${ }^{2}$ University of Zielona Góra, 9 Licealna Str., 65-417 Zielona Góra, Poland
}

\begin{abstract}
The article deals with the analysis of situations of uncertainty in various spheres of modern society life that have arisen as a result of the rapid development of digital technologies; virtualization of many components of modern human life; the increasing role of visual information in communication; drastic changes in the labor market, in intellectual practices and the formation of new requirements for the education and vocational training system. We have proposed to analyze such situations using the principle of "incomplete comprehension of object", and we carried out a consideration of its content and indicated its methodological role. To our mind, one of the most important features of this principle is the focus on a set of ambiguous, non-obvious links between the internal elements of an object, as well as during its interactions with agents of external influence in a situation of uncertainty. Besides, we examined its heuristic and predictive capabilities using examples of analysis of specific typical situations in various spheres of social life, primarily related to the labor market and education in a digital culture.
\end{abstract}

\section{Introduction}

In the context of Industry 4.0 and the rapid development of digital culture, a person is required to quickly and efficiently adapt to new ICT in production and everyday life $[1,2]$, professional orientation of a person, starting with junior and middle, and not only senior school age [3] education or self-education through massive open online courses [4-7], training in general secondary education institutions [8-11]. However, the development of digital technologies and their mass distribution leads not only to positive changes in the material sphere of human life, but also to some inconveniences and risks $[12,13]$. We are talking not only about the risks for an individual, but also about the prospects for the development of society and the future of humanity as a whole.

One of the important problems is the dramatic changes in the labor market in many countries, and this situation has obvious features of the object of analysis with many unknown qualities [14-16]. To develop an effective strategy for adapting to it, it is necessary to take into account extremely large amount of factors influencing such a situation, i.e. its determinants and conditions. Some countries are considering introducing an unconditional basic income, for example in Finland; reduction of working time to a 6-hour working day or introduction of a working week shortened to 3-4 days (Australia, Sweden) [17]. But there

\footnotetext{
*e-mail: polishchuk.o.p.2015@gmail.com

**e-mail: miller-melnik@ukr.net

***e-mail: irenevik@i.ua

****e-mail: r.sapenko@ifiluz.zgora.pl

†e-mail: bwtrocha@gmail.com
}

are other equally important problems associated with digital culture. A person is not like clockwork that can be complicated and complicated: here it is enough to recall the answer of the Swedish queen Christina to her opponents about their enthusiasm for the development of technology, the first machines and machine production in the modern era. The social consequences of the technical advances of the day were ambiguous. On the one hand, this is a sharp return on capital and an interest in machine production. But the sharp impoverishment of a significant number of people caused a change in the way of life and the needlessness of the previous number of workers, and social protests as a result (for example, there are the Luddite movement and the desire to destroy machines during the first industrial revolution on the part of workers in factories and artisans), on the other hand. However, the third important point of changes in the Europeans' way of life of that period was the change in the paradigm of teaching and education in general.

Since the late 1990s, and especially the last few years, we have been observing a similar situation, a kind of sociocultural "refrain" of radical, rapid and incomprehensible in terms of prospects for changing the way a person's daily life and the existence of society. This is happening due to the development of digital technologies and the digitalization of different spheres of life.

The object of this research is the content of the principle of "incomplete comprehension of object", its heuristic and predictive capabilities when considering risks and prospects for the development of modern society and a person in conditions of digital culture. The purpose of the study is to analyze the principle of "incomplete com- 
prehension of object" and its methodological value, as a component of the method of system analysis of complex phenomena, when considering modern phenomena, social processes and abrupt changes in various spheres of human life: production, everyday life and leisure, cultural development, education and upbringing.

\section{Review}

The problem of incomplete comprehension of objects, which is the important influence factors, is need for understanding the causes of the situation of uncertainties in modern people's life and its insecurity, anxiety and difficulties in adapting to the conditions of digital culture, virtualization and digital technologies. However, it is poorly studied. We pointed out the methodological value of "incomplete comprehension of object" principle when analyzing the phenomena of symbolic production and exchange during visual communication in the context of digital culture [18]. As a phenomenon of cognitive and epistemological order, André J. Abath considers the phenomenon of incomplete comprehension of object at the research process, linking it with the logic of understanding concepts by a person and partial interpretation of their content. The researcher pays special attention to the ratio of true knowledge about the research object with one of the false beliefs [19]. Some researchers point out the importance of studying the problem of incomplete knowledge [20, 21]. But Pedro Lima and João Gonzalez [22] point out the importance of studying the problem of programming robotic systems in conditions of incomplete comprehension of object. A group of Canadian scientists headed by Shahryar Rahnamayan, while analyzing the problem of incomplete comprehension of object, focused on the study of the phenomenon of recognition. Scientists suggest that when solving complex problems, "genetic algorithms" and operations on morphology, image processing procedures can be effectively used by a person [23]. According to researchers, the problem of recognition is important, given the importance of visualization in the life of contemporaries. At the same time, the authors indicate the presence of a "common reference sample, regardless of a priori knowledge" as a key characteristic of the recognition procedure [23]. It is important to point out, that researchers, while generally recognizing the value of analyzing the problem of incomplete comprehension of object, have not yet developed a unified model of its interpretation both in terms of content and practical significance.

Therefore, the principle of "incomplete comprehension of object" development within cognition process in terms of methodology is important and promising. This principle develops the prognostic field and heuristic potential of the system analysis of complex social phenomena and processes method, creating the prerequisites for effective orientation in the context of modern information and communication technologies.

\section{Discussion and results}

\subsection{A methodological setting of "incomplete comprehension of object" principle}

What does the principle of "incomplete comprehension of object" imply in the study of complex social objects, processes and phenomena? The importance of its development, as we assume as a hypothesis, is due to the fact that social life is:

1) a nonlinear system and therefore many links between its components are ambiguous, i.e., they cannot be considered "rigid", stable, constantly reproducing, especially during the period of rapid development of new technologies and materials;

2) it is important to consider the life of mankind and society as a dynamic phenomenon, some of the causes and consequences of which are implicit in perception, and therefore difficult to understand, interpret, but their ignorance or not paying proper attention to them can lead to social destruction, instability, regression;

3) there are cognitive limits in social cognition, individual learning, vocational education, when it comes to rapid changes in the way of life;

4) ideological attitudes and the value sphere of life, both of an individual and group, are more conservative, stable elements of a person's spiritual life rather than his knowledge in the professional sphere.

We want to draw attention to the emphasis which this principle is used for. According to it within cognition process it is necessary to identify and take into account the totality of ambiguous links between the internal elements of the object, as well as in its interaction with agents of external influence. It's them in their totality that constitute the field of uncertainty in the existence of complex objects (due to the number of components, a variety of external factors of influence, constant variability of elements or / and factors of influence). Such connections do not have a "hard" manifestation, i.e., they are probabilistic by nature (or at least are perceived to be ones).

Secondly, it is impossible to form an idea of the qualitative parameters of something or the properties of someone, based only on quantitative data. Of course, when trying to comprehend any social object, it's important to focus on the representativeness (typicality) of the observed events, phenomena, processes in everyday life.

Thirdly, the principle of "incomplete comprehension of object" implies the value of focusing attention on "agents of unusual reaction" to situations with a wide range of consequences. We believe that it is not necessary, of course, to detect the latter according to the principle of "grid in the sea", on the contrary, it is necessary to look for people or phenomena (processes, variables) which can be considered just atypical, unlike others, and they are quite striking. To our mind, in conditions of digital culture, "agents of an unusual reaction" are people whose reaction (primarily sensual) to what is happening in typical situations is unusual, unlike the reactions of other people. 
As an example, such a situation can be working with text, when word recognition is one of the first moments of its understanding. However, in addition to defining their meaning, there can be another level of human work on the text. Steve Mckee stated that reading is more than letter recognition, but involves determining meaning and context: "Direct or indirect connections are made between the information. As the information is processed, comprehension is developed. ... Meaning is assigned by using prior knowledge, looking at discourse structure, and context" [17]. To our mind, an unusual reaction of a person when perceiving a text is his attention to the context or indirect links between blocks of information. It doesn't matter which text it is (descriptive, narrative, explanatory, or argumentation). Moreover, not each of "poor comprehenders" [24] is, as we consider, an "agent of unusual reaction" to new information. In this case, the age or education of a person does not matter; it is his reaction to the interaction of basic (explicit) information and context (implicit) information in the text that is important. In other words, an important point is a person's ability (innate or arising from learning and training) to pay attention to the context of the situation or the implicit information contained in the text, message.

To our mind, paying attention to "agents of an unusual reaction" makes it possible, by analogy, to compare the typical with the atypical and identify both necessary and variable moments (properties, connections, elements of the external or internal structure) of an object that is important for analysis and discourse.

Therefore, such a methodological setting of "incomplete comprehension of object" principle will be useful when considering stochastic processes in modern society and studying any object: abrupt changes in the conditions of everyday life as a consequence of the increasing importance of digital technologies, the strengthening the role of visualization and symbolization of thinking in information processes, rapid modernization of some spheres, etc. (For example, the emergence of innovative digital programs, accordingly, causes necessity of rapid development of new training programs, and then you need to quickly understand the value of the latter).

Fourthly, the principle of "incomplete comprehension of object" directs the researcher not only pay attention to "agents of external influence" (based on analytical analysis). In such a case it is important to analyze the value and worldview basis of the life of "agents of an unusual reaction" (through observation, phenomenological reduction, and experiment). In our opinion, it is necessary to pay attention to the following point. First of all, it is important to understand how coherent their reaction to what is happening is, in order to reveal a tendency towards similarity in emotional and sensory responses, assessments, and actions. And if such a tendency is recorded, then one can try to simulate a general portrait or several of its profiles among "agents of unusual reaction". On its basis, it is important to identify not only latent motives for activity, intentions, incentives and preferences, fears or complexes, but also a group of social irritants as objects of hostility / sympathy.
The latter will be of the greatest interest, since they make it possible to identify implicit factors of influence during a sharp change in the way of everyday life, and this is precisely what is happening now with the massive introduction of digital technologies, virtualization of the life of society and the requirements for continuous education and self-education of workers. And the main reason for the latter is the constant updating of software, development of new devices and materials, means of communication and communication, etc.

In other words, it is important to focus the locus of attention of the researcher, mainly, not on positive moments, but on negative experience, primarily in human sensory reactions to novelty and innovation.

After all, the rejection of the requirement of constant software updates for office equipment or the acquisition of more and more new phone models is also a human reaction to the excessive fascination of modern society with "technical toys". It is not necessarily determined by its inability to quickly learn new technologies. On the contrary, such a reaction may be a manifestation of an extremely critical understanding that he does not need to follow fashion for a new trend and a sober calculation of his expenses.

\subsection{The practical implementation of "incomplete comprehension of object" principle}

The principle of "incomplete comprehension of object" focuses on the system analysis of complex objects in the context of their rapid transformation. It also expands the possibilities of modeling social objects. The principle of "incomplete comprehension of object" using for analysis makes it possible to adequately model the situation, one of the main features of which is uncertainty in understanding the determinants and conditions of its existence, the prospects for its development, etc. Uncertainty leads to different assessments of such situations on the part of contemporaries: understanding / misunderstanding, acceptance / rejection (depending on their personal understanding and preferences, as well as the influence of collective experience).

The main reason for the uncertainty in people's life in the conditions of Industry 4.0 and the anxiety caused by it is associated with the pressure of digital technologies and artificial intelligence on a person which is made by means of computational methods and information technologies. That makes some people change their professional specialty, typical for routine functions and jobs [25]. As a result of it modeling professional growth is based on intellectual analysis helps to discover more attractive activities, develop creative abilities [26]. For that reason, in a rapidly changing environment, only adequate proposals of educational institutions will help professionals effectively develop their careers [27]. Therefore, the practical implementation of the study, first of all, concerns the assessment of the professional orientation of modern youth, since Industry 4.0 technologies determine the rapid transformation of the labor market and new requirements for general and vocational training, as well as create new conditions for 
learning (massive open online courses [4]; cloud technologies and cloud services [28]; game simulators [29]). It should be noted that the changes concern various professional areas.

For example, the admission of a young person to the university depends on many factors, as well as his successful education or, conversely, unsuccessful performance results, and besides, his admission and graduation from this institution are mediated by many variable factors. These factors become especially uncertain in the absence of social stability for various reasons.

We point out that, such external factors are now distance learning for pupils and students thanks to the Zoom platform because of the Covid-19 pandemic (factor a), as well as a greater opportunity for their access to products of popular art and culture due to digital technologies (factor b). Both factors simultaneously influence the motivation to learn (goal a) and motivation for fun (goal b), including using the time allocated for learning and self-study (goal c). But the second of them was known and could be taken into account, while the first factor is completely unexpected and therefore unpredictable in its consequences at the moment. However, their simultaneous existence can lead to a spectrum of consequences, and they will be unequal for influence in different groups and in different countries. Then the "fan of possibilities" for the development of a social object becomes even more difficult to analyze. Situation 1: factor a can significantly reduce the influence of factor $b$ (for example, through the need to spend more time on preparation and self-preparation); situation 2: factor a significantly enhances factor $b$ (isolation from the collective has a depressive influence on a person and, as a result, his desire to acquire knowledge decreases and his desire to have fun increases, and modern popular culture gives him such an opportunity); situation 3: factor a will not affect factor $b$, etc. Besides, the existence of an electronic scientific / popular science book or Web book (factor c) is also a factor of influence in relation to factor a; e-book of fictional content (factor d). Such virtual objects are popular among modern youth. Therefore, factor $\mathrm{c}$ and factor $\mathrm{d}$ will also affect goal a (along the way, they will affect both goal $\mathrm{b}$ and goal c). Although they are not the main factors of influence, i.e. these are not obvious factors.

For other example, research made by Dell Technologies Research shows that 78 percents of workers across businesses consider digitalization to be a threat to them now and in the future; $45 \%$ of respondents are confident that their enterprises in the next 3-5 years will no longer be able to compete through digital technologies in production and communication; only 5 percents of enterprises are now leaders in the digitalization of production [1]. In modern society we now are already observing not only a decline in the popularity of many professions, but also a change in the professional active in many spheres of society. So, in the Scandinavian countries at least $90 \%$ of banking transactions are carried out online, and this leads to a drop in interest in the profession of a bank employee and a sharp decrease in the number of employees in this area. Therefore, the profession of a bank employee, which not long ago gave a person a stable and sufficiently high income and ensured social prestige, will lose its attractiveness for young people. In November 2020, Juniper Research indicated that the number of mobile users by 2025 will increase from 980 million (current year period) to 1.2 billion [14]. According to Juniper Research, this means that in the next 5 years, at least 30 percents of users in this market will only use financial services online. (We believe that the group represents those who are able to quickly adapt to the situation, i.e., "agents of unusual reaction" of the first type, adherents of digital culture). How correct this forecast is? It should be mentioned that the forecast doesn't take into account the conservatism of human thinking, unwillingness to learn innovative and rapidly changing digital technologies, etc. Besides, the rapid development of technology causes dramatic changes in the usual way of life, the emergence of new professions and the disappearance of other ones; as a result, we face the loss of jobs by many people, impoverishment and social protests. (We should remind, that during Industry 1 in England, the industry began to actively develop as a result of which many peasants lost the land, the mass pauperization of the population took place, "workhouses" appeared, the Luddite movement started and there appeared a saying "Sheep began to eat people". As the illustration to those processes, we can name the paintings of an eyewitness, Hogarth "Gin Lane" and others. At the same time during Industry 1 positive processes started: the development of science, emergence of the profession of a scientist, teaching and tutoring got popular). We consider, in order to develop an adequate forecast of the relevance of future profession, it is important to pay thorough attention to the "agents of an unusual reaction" of the second type, opponents of digital technologies. Only in this case we will be able to adequately assess the prospects for the development of this market and the social processes associated with it.

\section{Conclusion}

We want to pay attention to the excessive intellectual and emotional load of a modern person from new, digital technologies and many visual signals, messages that we have to deal with on a daily basis when performing professional duties, in everyday life, entertainment, education, and the like. As a result, people have many problems, first of all, chronic fatigue, self-doubt, aggressive behavior in society. In addition, the global digital sphere makes it necessary for a person to constantly learn throughout his life. The conditions for the rapid transformation of digital technologies and the dynamics of changes as a result of this in many spheres of society make it urgent to develop principles for the analysis of complex objects in order to form adequate forecasts and recommendations necessary to minimize destruction in social life. The principle of "incomplete comprehension of object" is able to fulfill this methodological heuristic and predictive role.

It seems to be important for orienting the researcher in the discourse of complex phenomena that occur in a digital society. One of their important features is uncertainty, especially in terms of development. But uncertainty is also 
recorded in the understanding / misunderstanding, acceptance / rejection of these phenomena by modern people and social groups.

The principle of "incomplete comprehension of object" focuses the attention of the researcher precisely on the uncertainty of the situation of the existence of complex objects in the conditions of their rapid changes. The principle of "incomplete comprehension of object" expands the capabilities of the system analysis method and modeling of social objects, therefore this principle will be useful in assessing the Industry 4.0 phenomenon and the rapid development of digital culture, as well as for developing medium-term and long-term forecasts in the sphere of material, social, and spiritual life of a person.

The complexity of understanding modern social processes is associated with their increased fragmentation, mosaicism and variability. This became especially evident in the situation of the Covid-19 pandemic and life under quarantine conditions, when the rules of human behavior changed significantly. Particularly alarming is the situation that has developed in the field of education, when children, adolescents and young people are forced to stay in atypical conditions for obtaining the necessary knowledge. Indeed, now in many countries distance learning is taking place thanks to new information and communication technologies, massive open online courses, game simulator, etc. are used. In fact, we have cardinal changes, even a breakdown of the existing intellectual practices and traditions of education, developed not only over the past decades, but in general since the period of the New Time and its Enlightenment project. The use of the principle of "incomplete comprehension of object" makes it possible to quickly assess the impact of this situation on their inner world, motivation for learning and the level of knowledge of our contemporaries.

\section{References}

[1] Dell Technologies Research: 78\% of Businesses Feel Threatened by Digital Start-ups (2020), https://corporate. delltechnologies.com/ru-ua/newsroom/ unveiling-the-digital-transformation-index . htm

[2] Y. Vorontsova, A. Arakelyan, V. Baranov, Wisdom 15, 68 (2020)

[3] M. Ehl, Aspen Review (2017)

[4] Z. Seidametova, CEUR Workshop Proceedings 2104, 462 (2018)

[5] L. Panchenko, I. Muzyka, CEUR Workshop Proceedings 2547, 168 (2020)

[6] M. Syvyi, O. Mazbayev, O. Varakuta, N. Panteleeva, O. Bondarenko, CEUR Workshop Proceedings 2731, 369 (2020)

[7] T. Vakaliuk, A. Yefimenko, V. Bolotina, Y. Bailiuk, O. Pokotylo, S. Didkivska, CEUR Workshop Proceedings 2732, 665 (2020)
[8] G. Almerich, N. Orellana, J. Suarez-Rodriguez, I. Diaz-Garcia, Computers \& Education 100, 110 (2016)

[9] S. Biswas, How can technology help in education? (2011), http: //www. cloudtweaks. com/2011/02/ how-can-cloud-computing-help-in-education

[10] C. Costa, H. Alvelos, L. Teixeira, Procedia Technology 5, 334- (2012)

[11] M. Piccarozzi, B. Aquilani, C. Gatti, Sustainability 10, 2 (2018)

[12] Systemic risk of modelling in insurance. did your model tell you all models are wrong? (2015), https://tigerrisk. com/wp-content/uploads/2018/10/ SystemicRisksofModellingFINALV3.pdf

[13] G. Bansal, F.M. Zahedi, D. Gefen, Information and Management 53, 1-21 (2016)

[14] M. Menin, 5 emerging consulting professions for the new economy (2020), https://riverflex.com/5emerging-consulting-professions-for-the-neweconomy

[15] World Economic Forum, The future of jobs report 2020 (2020), http://www3. weforum.org/docs/ WEF_Future_of_Jobs_2020.pdf

[16] McKinsey Global Institute, What's next for remote work: An analysis of 2,000 tasks, 800 jobs, and nine countries (2020), https://tinyurl.com/ uby $83 \mathrm{t} 3 \mathrm{~h}$

[17] S. Mckee, Language Testing in Asia 2, 45 (2012)

[18] O. Polishchuk, I. Vitiuk, N. Kovtun, V. Fed, Wisdom 15, 45 (2020)

[19] A.J. Abath, Principia 24, 419 (2020)

[20] T. Eiter, W. Faber, N. Leone, G. Pfeifer, A. Polleres, Planning under Incomplete Knowledge, in Computational Logic - CL 2000, edited by J. Lloyd, V. Dahl, U. Furbach, M. Kerber, K.K. Lau, C. Palamidessi, L.M. Pereira, Y. Sagiv, P.J. Stuckey (Springer Berlin Heidelberg, Berlin, Heidelberg, 2000), pp. 807-821, ISBN 978-3-540-44957-7

[21] J. Ferguson, M. Gardner, H. Hajishirzi, T. Khot, P. Dasigi, IIRC: A Dataset of Incomplete Information Reading Comprehension Questions, in Proceedings of the 2020 Conference on Empirical Methods in Natural Language Processing (EMNLP) (Association for Computational Linguistics, Online, 2020), pp. 1137-1147, https://www.aclweb. org/anthology/2020.emnlp-main. 86

[22] J. Gonçalves, P. Lima, Grasp Planning with Incomplete Knowledge About the Object to be Grasped, in 2019 IEEE International Conference on Autonomous Robot Systems and Competitions (ICARSC) (2019), pp. 1-6

[23] S. Rahnamayan, H.R. Tizhoosh, M.A. Salama, WSEAS Transactions on Systems 4, 1725 (2005)

[24] J. Kirby, Reading comprehension: Its nature and development (2007), http://web. archive.org/web/20101126123217/http: 
//literacyencyclopedia.ca/index $\cdot$ php?fa= items. show\&topic $I d=227$

[25] V.I. Sigov, S.A. Uvarov, N.N. Pokrovskaia, Neurofuzzy tools in studying social management, in 2017 IEEE II International Conference on Control in Technical Systems (CTS) (2017), pp. 216-219

[26] M.Y. Ababkova, N.N. Pokrovskaia, I.R. Trostinskaya, The European Proceedings of Social \& Be- havioural Sciences (EpSBS) XXXV, 10 (2018)

[27] N. Pokrovskaia, Y. Margulyan, A. Bulatetskaia, S. Snisarenko, Wisdom 14, 69- (2020)

[28] M. Volikova, T. Armash, Y. Yechkalo, V. Zaselskiy, CEUR Workshop Proceedings 2433, 486 (2019)

[29] T. Vakaliuk, V. Kontsedailo, D. Antoniuk, O. Korotun, I. Mintii, A. Pikilnyak, CEUR Workshop Proceedings 2547, 66 (2020) 